\title{
Detector for terahertz applications based on a serpentine array of integrated GaAs/InGaAs/AlGaAs-field-effect transistors
}

\author{
D.M. Yermolaev ${ }^{1,2}$, I. Khmyrova ${ }^{3}$ E. A. Polushkin ${ }^{1}$, A. V. Kovalchuk ${ }^{1}$, \\ V.I. Gavrilenko ${ }^{4}$, K.V. Maremyanin ${ }^{4}$, N.A. Maleev ${ }^{5}$, V.M. Ustinov ${ }^{5}$, V.E. Zemlyakov ${ }^{6}$, \\ V.A. Bespalov ${ }^{6}$, V.I. Egorkin ${ }^{6}$, V.V. Popov ${ }^{7}$, and S.Yu.Shapoval ${ }^{1}$ \\ ${ }^{1}$ Institute of Microelectronic Technology and High-Purity Materials, 14243 Chernogolovka, Russia \\ ${ }^{2}$ JSC Russian Space System, Moscow, 111250, Russia \\ ${ }^{3}$ University of Aizu, Aizu-Wakamatsu, Fukushima, 965-8580, Japan \\ ${ }^{4}$ Institute for Physics of Microstructures, 603950 Nizhny Novgorod, Russia \\ ${ }^{5}$ Ioffe Physical Technical Institute, 194021 St. Petersburg, Russia \\ ${ }^{6}$ National Research University of Electronic Technology, 124498 Zelenograd, Russia \\ ${ }^{7}$ Kotelnikov Institute of Radio Engineering and Electronics (Saratov Branch), 410019 Saratov, Russia
}

\begin{abstract}
Performance of a detector based on AlGaAs/InGaAs/GaAs-material system was studied. The detector was comprised of large serpentine array of high-electron mobility transistors (HEMTs) connected in series. The floating drain contact of each transistor (except the last one) served as a source for the next one. Detection of terahertz $(\mathrm{THz})$ radiation was based on the excitation of electron plasma oscillations in the HEMT's channel. The peculiarities of $\mathrm{THz}$ response of the detector in question including an enhanced noise-equivalent power were demonstrated. *
\end{abstract}

Keywords - FET array, terahertz radiation, plasma waves, floating electrode

\section{INTRODUCTION}

Detection and generation of terahertz $(\mathrm{THz})$ radiation based on excitation of plasma wave oscillations in the two-dimensional electron gas (2DEG) channel of field-effect transistor (FET)-like structures were theoretically predicted by $\mathrm{M}$. Dyakonov and M. Shur [1]. In recent years the achieved responsivity and noise equivalent power (NEP) of such detectors have approached the values meeting the demands of practical applications [2-7]. Main designs of plasma wave $\mathrm{THz}$ detectors include single transistors with the antenna $[3,4,7]$, single transistors with grating/interdigitated gate $[2,8,9]$ and several transistors connected by external wires or integrated on a chip [5,6]. Lateral dimensions of most highly sensitive detectors are much smaller than the half-wavelength of $\mathrm{THz}$ radiation $[5,6]$ and in an experiment one usually deals with a single detector surrounded by wiring. Indeed, the performance of such detectors arranged in the focal plane array (FPA) as well as the effect of external wiring are of great interest.

In a regular quasi-optical systems radiation beam can be focused on a spot with a diameter of halfwavelength covering several detectors in the FPA. Therefore, the radiated power will be distributed

*ISBN 978-80-261-0641-8, (C)University of West Bohemia, 2017 among neighboring detectors so that the power per one detector in the FPA will be proportional to the ratio of the detector area to the area of the irradiated spot which will affect the responsivity as well. We should also keep in mind the contribution of irradiated wiring which is different in the case of a single detector and FPA.

In the study of a FPA with $3 \times 5$ pixels [7] each pixel consisted of a transistor with patch antenna and integrated amplifier and had dimensions $200 \times 150$ $\mu \mathrm{m}^{2}$. At room temperature the detectors have demonstrated following performance: responsivity of about $70 \mathrm{kV} / \mathrm{W}$ with $43 \mathrm{~dB}$ voltage amplifier at $650 \mathrm{GHz}$, $\mathrm{NEP}=300 \mathrm{pW} / \sqrt{\mathrm{Hz}}$ at $30 \mathrm{kHz}$. A single detector with a log-periodic aerial antenna with diameter of $1.5 \mathrm{~mm}$ has been investigated in the frequency range 200-600 GHz at liquid helium temperature [10]. This detector has demonstrated a responsivity of $2.5 \mathrm{kV} / \mathrm{W}$ at excitation frequency of $240 \mathrm{GHz}$, its calculated NEP $100 \mathrm{pW} / \sqrt{\mathrm{Hz}}$ at $750 \mathrm{~Hz}$. Other configurations of transistors on a single chip have been also studied [2,5,6]. However, FPAs or single detectors with a size corresponding to radiation half-wavelength were not reported so far although the performance of detectors in the FPA as well as single detector of radiation half-wavelength dimensions are of great interest and worth to be investigated.

In this paper we present experimental results on $\mathrm{THz}$ response of a detector comprising of connected in series individual transistors which can be treated as FPA or as a single detector with dimensions of radiation half-wavelength.

\section{Detector FABricAtion}

$\mathrm{THz}$ detectors with GaAs/InGaAs/AlGaAs heterostructures on semi insulating GaAs substrate were fabricated. At room temperature electron mobility and concentration in the channel were $5900 \mathrm{~cm}^{2} / \mathrm{V} \times \mathrm{s}$ and $3 \times 10^{12} \mathrm{~cm}^{-2}$, respectively. The cap layer was $\mathrm{n}$ doped with $\mathrm{Si}$ up to $6 \times 10^{18} \mathrm{~cm}^{-3}$. Ohmic contacts 


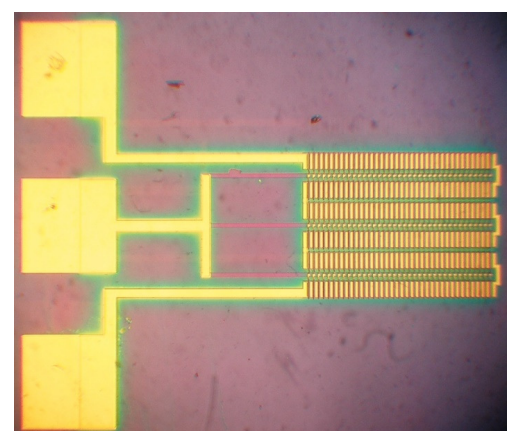

Fig. 1. Photo of the detector obtained with optical microscope.

(source, drain, gate bus) were formed by deposition of 30/10/40nm AuGe-Ni-Au layers. Gate metalization (metal-semiconductor contact) was made by $150 / 500$ nm Ti-Au layers. The substrate was thinned to $100 \mu \mathrm{m}$. Photo of the detector and schematics of the detector structure (top view) are shown in Figs. 1 and 2, respectively. The metal gate fingers are connected with gate bus via mesa to eliminate shunting of currents induced by $\mathrm{THz}$ radiation as proposed in [11]. In addition, the symmetry of the structure of transistors in each next row is inverted in respect to location of the metal strips of the gate. This changes the orientation of source-to-drain direction with respect to the direction of polarization of incident $\mathrm{THz}$ field as well as the direction of the rectified current. Such configuration promotes the flow of rectified currents from all rows of transistors in one direction. The detector has 6 rows with 37 transistors in each. The detection area is $300 \times 200 \mu \mathrm{m}^{2}$. The width of transistors' channels is $25 \mu \mathrm{m}$.

The mesa with the FET chain was mounted in the sample holder allowing to apply dc gate bias to the common gate pad and dc drain bias between the side (source and drain) pads of the FET chain. It should be mentioned that all contacts playing the roles of sources and drains except the first and the last pads are floating. Two monochromatic sources of radiation were used: backward wave oscillator (BWO) and radiofrequency (RF) generator. BWO was used as a source of sub- $\mathrm{THz}$ radiation in frequency range of 415-720 $\mathrm{GHz}$. Asymmetry in gate location in respect to ohmic contacts makes it possible to realize photovoltaic detection mode.

The RF generator has been set at a single frequency $143 \mathrm{GHz}$ with power in the maximum of $50 \mathrm{~mW}$. The output power was calibrated by the Deuterated Triglycine Sulphate (DTGS) pyroelectric detector, which is an authorized tool supplied with the BOMEM DAS.36 FTIR spectrometer. Terahertz radiation was mechanically chopped at a frequency $400 \mathrm{~Hz}$ and directed to the sample through an oversized hollow circular copper waveguide. Tapered end of the waveguide had an output aperture of $6 \mathrm{~mm}$ in diameter which fully covered the FETs array. Power attenuation in the oversized circular copper waveguide measured by the DTGS pyroelectric detector was about $10 \mathrm{~dB}$ in the frequency range of interest. The output aperture

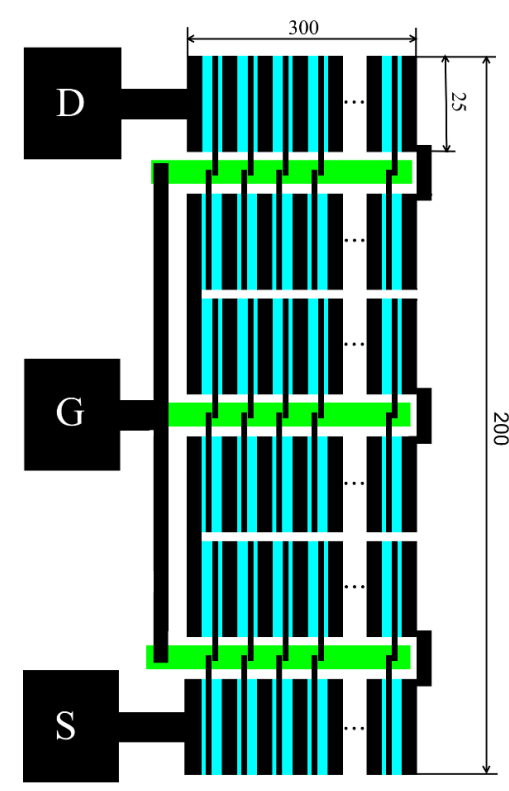

Fig. 2. Schematics of the detector structure (top view). Dimensions are in $\mu \mathrm{m}$.

of this waveguide was placed just above the sample so that the incident $\mathrm{THz}$ power was distributed fairly homogeneous over the sample area. By estimate, the power of $\mathrm{THz}$ radiation incident on the sample area of $200 \times 300 \mu \mathrm{m}^{2}$ was about $210 \mathrm{nW}$ for BWO and 11 $\mu \mathrm{W}$ for RF generator. The electric field of the incident $\mathrm{THz}$ wave was polarized across the FET-gate fingers. The source pad was grounded and the gate voltage was applied between the gate and source pads. The THz photovoltage $U_{p h}$ was measured at the drain pad. The photovoltage signal measurements were performed by standard lock-in technique. Static current-voltage characteristics of the FET array is shown in Fig. 3. The channel depletion threshold voltage was about $U_{t h}=1.3 \mathrm{~V}$.

\section{OBTAINED RESULTS AND DISCUSSION}

Measurement of electromotive force (EMF) by BWO was carried out at liquid helium temperature for normally incident radiation with frequency $f \approx 0.6$ $\mathrm{THz}$ (wavelength $\lambda=500 \mu \mathrm{m}$ ). Experimentally measured EMF is shown in Fig. 4. The maximum EMF is about $300 \mu \mathrm{V}$ at $U_{g s} \approx 1.15 \mathrm{~V}$. The shape of this curve is typical for nonresonant $\mathrm{THz}$ response. As long as the

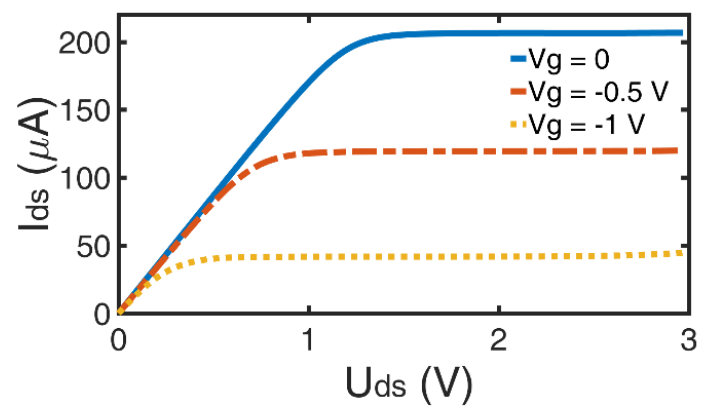

Fig. 3. Current-voltage characteristics of detector at $4.2 \mathrm{~K}$. 


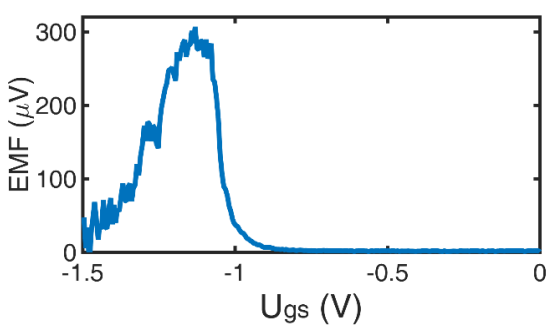

Fig. 4. Experimentally measured EMF at a frequency $f \approx 0.6$ THz.

detector has asymmetrical structure the response can be measured without a biased current. The responsivity can be calculated as $R_{e s}=E M F / P$, where $P$ is power of radiation incident on the sample surface. Estimated maximum responsivity is about $R_{e s} \approx 1.4$ $\mathrm{kV} / \mathrm{W}$. Another important characteristics is signal-tonoise ratio. NEP of the detector was evaluated using the following formula:

$$
N E P=\sqrt{\frac{4 k_{B} R_{S D}}{R_{e s}}},
$$

where $k_{B}$ is the Boltzmanns constant, $R_{S D}$ is the source-drain resistance. The dependence of NEP versus $U_{g s}$ is shown in Fig. 5.

The minimum of the NEP is about $1 \mathrm{pW} / \sqrt{H z}$ at $U_{g s} \approx 1.1 \mathrm{~V}$. The obtained results are quite similar to those presented in [10] which gives a ground to consider both types of detectors pretty close to each other. However, the proposed detector with serpentine array of FETs may have some advantages in terms of the NEP and responsivity. It is worth to mention the quadratic dependence of the latter on frequency in the $\mathrm{THz}$ range [12]. It should be emphasized also that two order increase in the responsivity of the detector presented in [10] was achieved by attachment of the focusing lens [4]. The resistance of the channel has a value $10^{5} \mathrm{Ohm}$ at the voltage $1.1 \mathrm{~V}$. Therefore there is a mismatch of the impedance of free space and the receiver that reduces the responsivity.

To evaluate the change of the response with the temperature we used more powerful source of radiation described in Section II. Experimental results are presented in Fig. 6.

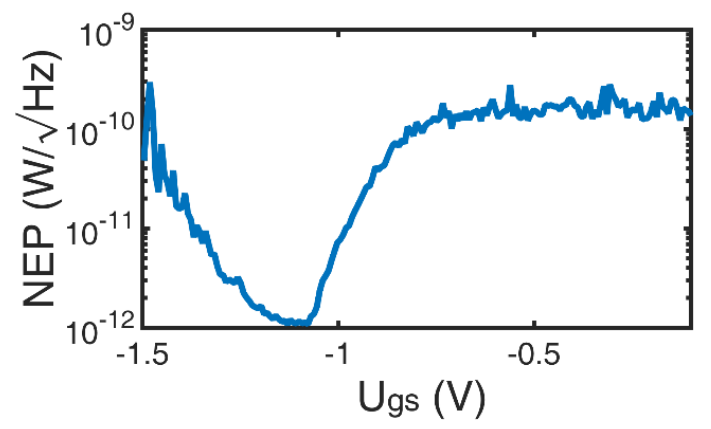

Fig. 5. NEP of the detector versus $U_{g s}$

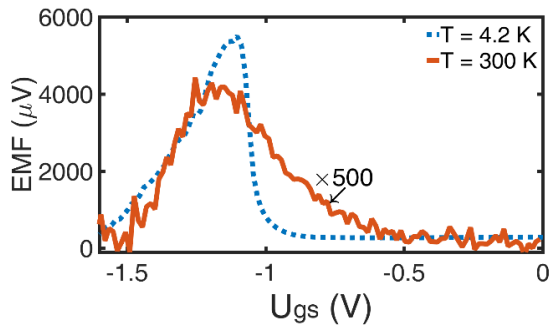

Fig. 6. EMF of the detector versus $U_{g s}$ at different temperatures $(4.2$ and $300 \mathrm{~K})$. Curve at room temperature is multiplied by 500 .

Due to different temperature ranges used in measurements there is data shortage for full scale comparison of the responses of the detector under investigation and those ones from Refs. [4,10]. However, it has been demonstrated that at $40 \mathrm{GHz}$ response of the detector with lateral dimensions several times smaller than radiation half-wavelength has dropped more than three orders in magnitude with the temperature [13]. Thus, the detectors with a size close to radiation half-wavelength can exhibit stable characteristics in a broader temperature range.

\section{CONCLUSIONS}

In conclusion, we fabricated a single-chip detector comprising of a large number of transistors connected in series, dimensions of the detector are close to half-wavelength of $\mathrm{THz}$ radiation to be detected. The obtained voltage responsivity is above $1.4 \mathrm{kV} / \mathrm{W}$ in the unbiased mode of the detector operation, the NEP of the detector is $10^{-12} \mathrm{~W} / \sqrt{\mathrm{Hz}}$ at $0.6 \mathrm{THz}$ and $4.2 \mathrm{~K}$. The proposed design is not optimal due to mismatch of the impedances of free space and the detector. It should be improved and the current design has a plenty of resources for that. Optimization of the detector with proposed configuration opens up new opportunities for further development of $\mathrm{THz}$ detectors for different applications.

\section{ACKNOWLEDGMENTS}

This work was supported by the Russian Federal Target Program Development of Electronic Component Base and Radio Electronics (Contract No. 14.427.11.0004) and Russian Academy of Sciences Program Fundamentals and Development of Sensor Nanoelectronics and Devices for Terahertz Frequency Ranges (No. 0070-2014-0006).

\section{REFERENCES}

[1] M. Dyakonov and M. Shur, "Detection, mixing and frequency multiplication of terahertz radiation by two-dimensional electronic fluid", IEEE Trans. Electr. Dev., vol. 43, pp. 380387 , 1996.

[2] T. Watanabe, S. Boubanga Tombet, Y.'Tanimoto, Y. Wang, H. Minamide, H. Ito, D. Fateev, V. Popov, D. 'Coquillat, W. Knap, "Ultrahigh sensitive plasmonic terahertz detector based on an asymmetric dual-grating gate HEMT structure", Solid State Electron., vol. 78, pp. 109114, 2012.

[3] F. Schuster D. Coquillat, H. Videlier, M. Sakowicz, F. Teppe, L. Dussopt, B. Giffard, T. Skotnicki, and W. Knap, "Broadband terahertz imaging with highly sensitive silicon CMOS detectors" Opt. Exp. vol. 19, pp. 7827-7832, 2011. 
[4] G. C. Dyer, S. Preu, G. R. Aizin, J. Mikalopas, A. D. Grine J. L. Reno, J. M. Hensley, N. Q. Vinh, A. C. Gossard, M. S. Sherwin, S. J. Allen, and E. A. Shaner, "Enhanced performance of resonant sub-terahertz detection in a plasmonic cavity", Appl. Phys. Lett., vol. 100, 083506, 2012.

[5] T. A. Elkhatib, V. Y. Kachorovskii, W. J. Stillman, D. B. Veksler, K. N. Salama, X.-C. Zhang, and M. S. Shur, "Enhanced plasma wave detection of terahertz radiation using multiple high electron-mobility transistors connected in series" IEEE Trans. Microwave Theory Tech., vol. 58, pp. 331-339, 2010.

[6] V. V. Popov, D. M. Yermolaev, K. V. Maremyanin, V. E. Zemlyakov, N. A. Maleev, V. I. Gavrilenko, V. A. Bespalov, V. I.'Yegorkin, V. M. Ustinov, and S. Yu. Shapoval "Detection of terahertz radiation by tightly concatenated InGaAs field-effect transistors integrated on a single chip", Appl. Phys. Lett., vol. 104, 163508, 2014.

[7] A. Lisauskas, U. Pfeiffer, E. Ojefors, P. H. Bolvar, D. Glaab, and H. G. Roskos, 'Rational design of high-responsivity detectors of terahertz radiation based on distributed self-mixing in silicon field-effect transistors" J. Appl.PHys., vol. 105, 114511 , 2009
[8] S. Boubanga-Tombet, Y. Tanimoto, A. Satou, T. Suemitsu, Y. Wang, H. Minamide, H. Ito, D. V. Fateev, V. V. Popov, and T. Otsuji, "Current-driven detection of terahertz radiation using a dual-grating-gate plasmonic detector", Appl. Phys. Lett., vol. 104, 262104, 2014.

[9] I. Khmyrova, R. Yamase, M. Fukuda, and N. Watanabe, "Analysis of terahertz plasma resonances in structures with two-dimensional elec tron systems periodically modulated by interdigitated gate", J. Appl. Phys., vol. 108, 074511, 2010.

[10] G. C. Dyer, N. Q. Vinh, S. J. Allen, G. R. Aizin, J. Mikalopas, J. L. Reno, and E. A. Shaner, "A terahertz plasmon cavity detector", Appl. Phys. Lett., vol. 97, 193507, 2010.

[11] D. M. Yermolayev, E. A. Polushkin, S. Yu. Shapoval, V. V. Popov, K. V. Maremyanin, V. I. Gavrilenko, N. A. Maleev, V. M. Ustinov, V. E. Zemlyakov, V. I. Yegorkin, V. A. Bespalov, A. V. Muravjov, S. L. Rumyantsev, and M. S. Shur, Detection of terahertz radiation by dense arrays of InGaAs transistors, Int. J. High Speed Electron. Syst. vol. 24, 1550002, 2015.

[12] M. Tonouchi, Cutting-edge terahertz technology, Nat. Photon., vol. 1, pp. 97105, 2007.

[13] V. M. Muravev and I. V. Kukushkin, Plasmonic detector/spectrometer of subterahertz radiation based on twodimensional electron system with embedded defect, Appl. Phys. Lett., vol. 100, 082102, 2012. 